\title{
Atom Interferometry tests of the isotropy of post-Newtonian gravity
}

\author{
Holger Müller ${ }^{1}$ * Sheng-wey Chiow ${ }^{1}$, Sven Herrmann ${ }^{1}$, and Steven Chu ${ }^{1,2}$ \\ ${ }^{1}$ Physics Department, 382 Via Pueblo Mall, Stanford, California 94305, U.S.A. \\ ${ }^{2}$ Lawrence Berkeley National Laboratory and Department of Physics, \\ University of California, Berkeley, 1 Cyclotron Road, Berkeley, California 94720, U.S.A. \\ Keng-Yeow Chung \\ Physics Dept., National University of Singapore, 2 Science Drive 3, Singapore 117542
}

(Dated: September 15, 2018)

\begin{abstract}
We present a test of the local Lorentz invariance of post-Newtonian gravity by monitoring Earth's gravity with a Mach-Zehnder atom interferometer that features a resolution of about $8 \times 10^{-9} \mathrm{~g} / \sqrt{\mathrm{Hz}}$, the highest reported thus far. Expressed within the standard model extension (SME) or Nordtvedt's anisotropic universe model, the analysis limits four coefficients describing anisotropic gravity at the ppb level and three others, for the first time, at the 10ppm level. Using the SME we explicitly demonstrate how the experiment actually compares the isotropy of gravity and electromagnetism.
\end{abstract}

PACS numbers: 03.75.Dg, 11.30.Cp, 11.30.Qc, 04.25.Nx

The description of gravitation by a dynamic geometry of space-time, Einstein's general relativity (GR), is based on the Einstein equivalence principle. This encompasses the universality of free fall (UFF), local position invariance (LPI), and local Lorentz invariance (LLI), which also underlies the non-gravitational standard model of particle physics. Attempts to unify GR and the standard model have failed so far. This suggests that one of their foundations might be violated at some level of precision [1]. So far, tests of the UFF and LPI have not identified violations [1]. LLI has been tested experimentally for many sectors of the standard model, such as for photons ('Maxwell sector'), electrons, protons, and neutrons [1, 2, 3]. No Lorentz violation has been identified, although the coverage of parameter space is still incomplete. Far less attention, however, has been paid to the LLI of the gravitational ('Einstein') sector, in spite of the pioneering work of Nordtvedt and Will in the 1970ies. Motivated by that fact that anisotropies arise in various theories of gravity other than GR [4], they have ruled out a Lorentz-violating anisotropy in gravity by searching for an anomalous time-dependence of the acceleration of free fall $g$ on Earth [4, 5, [6].

The success of GR and the standard model implies that any Lorentz violations are tiny. This and the relative weakness of gravity means that only exceptionally sensitive experiments can hope to detect Lorentz violation in gravity. A relatively recent addition to these is precision atom interferometry [7, 8]. This has been serving, for example, in measurements of the fine structure constant [9], $g$ [10] and its gradient [11], the Sagnac effect [12], and Newton's constant $G$ [13] with sensitivities that compare favorably with other state-of-the-art instruments. One reason for its outstanding precision is that the motion of neutral atoms can realize a freely falling frame to a

*Electronic address: holgerm@stanford.edu high accuracy and that this motion can be interrogated by laser radiation in a tremendously precise way. As a result, tests of post-Newtonian gravity with atom interferometry have been proposed that could rival or exceed the precision of classical ones [14].

Here, we report on a first step in this direction: We describe the highest resolution atomic gravimeter reported thus far [15]. We then analyze the influence of Lorentz violation in gravity. By explicitly including possible Lorentz violation in electrodynamics, we explicitly show how this (like any) isotropy test is actually a comparison of two sectors. Finally, we report a test of the LLI of post-Newtonian gravity by testing its isotropy.

Our experimental setup (Fig. 2) assembles about $10^{9}$ Cs atoms within $650 \mathrm{~ms}$ from a background vapor pressure of $\sim 10^{-9}$ mbar in a 3 -dimensional magneto-optical trap (3D-MOT). A moving optical molasses launch accelerates them vertically upwards to a $\sim 1-\mathrm{s}$ ballistic trajectory with a temperature of $1.2-2 \mu \mathrm{K}$. Raman sideband cooling in a co-moving optical lattice results in $\sim 3 \times 10^{8}$ atoms in the $F=3, m_{F}=3$ state at a (3D) temperature of $150 \mathrm{nK}$ that form a cloud of roughly $3 \mathrm{~mm}^{2}$ area [17]. A sudden change in the magnetic field followed by a $120-\mu$ s microwave pulse transfers $\sim 20 \%$ of them into the $F=4, m_{F}=0$ state. Atoms left over in the $F=3$ state are then cleared away using a resonant laser pulse. A solenoid generates a small magnetic bias field to set the quantization axis.

Afterwards, a ' $\pi / 2$ ' pulse of counterpropagating laser beams, overlapped with the trajectory of the atoms, transfers the atoms into a superposition of the $F=3$ and $F=4$ hyperfine ground states by a two-photon Raman transition (Fig. 1, left). These states move vertically relative to each other because of the momentum of two photons transferred by the laser radiation. After a time $T \simeq 0.4 \mathrm{~s}, \mathrm{a}$ ' $\pi$ ' pulse interchanges the $F=3$ and 4 states, which afterwards move towards each other. After another $T$, a final $\pi / 2$ pulse recombines the paths to form 

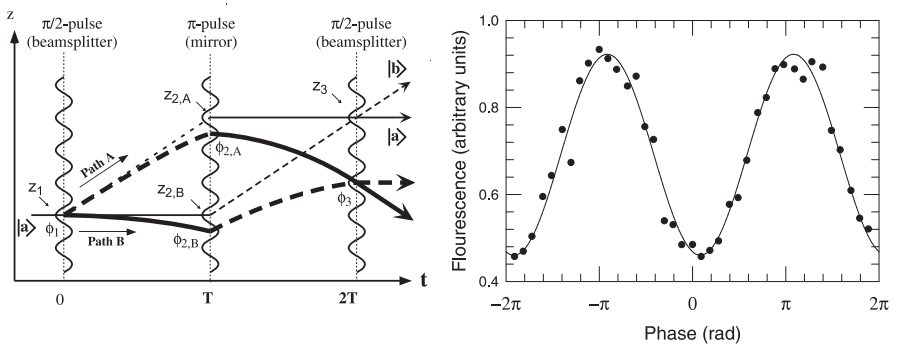

FIG. 1: Left: Schematic of a Mach Zehnder atom interferometer. In our experiment, a $\pi$ pulse takes about $100 \mu$ s. Right: typical fringe obtained in our experiment

an interferometer.

The off-resonant Raman pulses are generated by two extended cavity diode lasers based on $100-\mathrm{mW}$ laser diodes SDL-5411. The first is frequency stabilized ('locked') by Doppler-free frequency modulation (FM) spectroscopy to a Cs vapor cell. It arrives at the interferometer with a detuning of $-1030 \mathrm{MHz}$ from the $6 S_{1 / 2}, F=3 \rightarrow 6 P_{3 / 2}, F^{\prime}=4$ transition (at $852 \mathrm{~nm}$ wavelength) in Cs. The second one is phase locked to the first one with a frequency difference close to the hyperfine splitting of $\simeq 9192 \mathrm{MHz}$, referenced to a LORAN-C frequency standard. $20 \mathrm{~mW}$ of each laser are transmitted to the experiment via a common single-mode, polarization maintaining optical fiber. The beams are then switched and intensity-controlled by an acousto-optical modulator (Isomet 1205), expanded to about $2.5 \mathrm{~cm}$, and pass the vacuum chamber with linear polarization. Retroreflection on top of it with two passes through a quarterwave retardation plate forms a lin $\perp$ lin polarized counterpropagation geometry.

The matter waves in both interferometer paths acquire a phase difference $\phi$. The contribution of the free evolution, given by the classical action $S_{C l} / \hbar$ vanishes for a constant $g$. However gravity shifts downwards the location at which the paths interact with the light by $\Delta z=-g t^{2} / 2$, where $t$ denotes time and $z$ the vertical coordinate. This gives rise to a phase difference (assuming the UFF) [10]

$$
\phi=k_{\mathrm{eff}} g T^{2}-\phi_{L}
$$

where $\phi_{L}=\phi_{1}-2 \phi_{2}+\phi_{3}$ is given by the phases $\phi_{1-3}$ of the laser fields at $z=0$. To high accuracy, the laser radiation can be modelled as a plane wave, which results in an effective wavevector $k_{\text {eff }}=2 k=2 \omega / c$. For our experiment, $\phi \simeq 2.3 \times 10^{7} \mathrm{rad}$. To measure it, we adjust $\phi_{L}$ such that $\phi=0$, which corresponds to the center of the interference pattern. This can be done by using $\phi_{L}=r T^{2}$, i.e. by ramping the difference frequency at a rate $r$ or a step-wise approximation of such a ramp.

Both the $F=3$ and 4 interferometer outputs are detected by flourescence detection with a Hamamatsu R943-02 photomultiplier tube (PMT). Normalization of the signals takes out atom number variations.

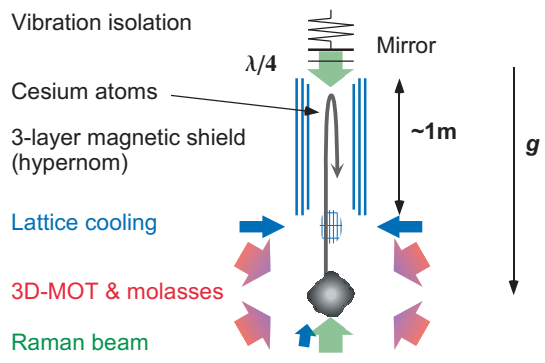

FIG. 2: Setup. $\lambda / 4 ; 1 / 4$-wave retardation plate. Vibrations of the top mirror are reduced to below $5 \times 10^{-9} \mathrm{~g} / \sqrt{\mathrm{Hz}}$ in a frequency range of $0.1-10 \mathrm{~Hz}$ by a sophisticated active vibration isolator [16].

Fig. 1 (right) shows a typical fringe with a pulse separation time of $T=400 \mathrm{~ms}$, taken with 40 launches that take $75 \mathrm{~s}$ total. A sinewave-fit has a phase uncertainty of $0.031 \mathrm{rad}$, and determines $g$ to an uncertainty of $\sim 1.3 \times 10^{-9} \mathrm{~g}$. This corresponds to $11 \times 10^{-9} \mathrm{~g} / \sqrt{\mathrm{Hz}}$. An improved short-term resolution of $8 \times 10^{-9} \mathrm{~g} / \sqrt{\mathrm{Hz}}$ can be reached by taking data at the $50 \%$ points of the fringes only. However, as this method is more sensitive to systematic effects such as drift of the PMT sensitivity [10], this approach was not followed. Our resolution is more than 3 times better than the best previously reported [10]. It also surpasses the best classical absolute gravimeter, the FG-5 falling corner cube gravimeter, by a factor of about 20 .

The notion that gravity might depend on the direction of the separation $\vec{r}$ could be described in very simple terms. For this work, however, we want to use a model that is as general as possible on the one hand and compatible with accepted principles that underlie the standard model and gravitational theory on the other hand. Two such models suggest themselves, Nordtvedt's anisotropic universe model [4] and the standard model extension (SME) [2, 18]. The SME starts from a Lagrangian formulation of the standard model and gravity, adding general Lorentz violating terms that can be formed from the fields and tensors. For the gravitational fields present on Earth, a post-Newtonian approximation is justified. For two masses $M$ and $m$, separated by $\vec{r}$, where $M$ is assumed to be at rest, the Lagrangian for the gravitational interaction in the SME is [18]

$$
\begin{gathered}
\mathcal{L}=\frac{1}{2} m v^{2}+G \frac{M m}{2 r}\left(2+3 \bar{s}^{00}\right. \\
\left.+\bar{s}^{j k} \hat{r}^{j} \hat{r}^{k}-3 \bar{s}^{0 j} v^{j}-\bar{s}^{0 j} \hat{r}^{j} v^{k} \hat{r}^{k}\right) .
\end{gathered}
$$

The indices $j, k$ denote the spatial coordinates, $\vec{v}$ the relative velocity, and $\hat{r}=\vec{r} / r . \bar{s}^{\mu \nu}=\bar{s}^{\nu \mu}$ specifies Lorentz violation in gravity. The two-body Lagrangian of the anisotropic universe model is similar, but $\bar{s}^{00}=0$ and the coefficients of the $v^{j}$ and the $\hat{r}^{j} v^{k} \hat{r}^{k}$ terms are independent of each other. The equation of motion (simplified by using $v \ll 1$ and neglecting constant as well as 
horizontal accelerations) reads

$$
\ddot{r}^{l}+\hat{r}^{l} \frac{G M}{2 r^{2}}\left(2+\bar{s}^{j k} \hat{r}^{j} \hat{r}^{k}\right)=0
$$

where the coefficient of $\hat{r}^{l}$ gives the modified acceleration of free fall.

One outstanding feature of atom interferometry is the relative simplicity of the underlying theoretical assumptions, which can be traced to its relying on nonrelativistic single-particle effects. This allows us to analyze the experiment without assuming the LLI of the Maxwell sector. We therefore determine $k_{\text {eff }}$ in Eq. (1) from the dispersion relation for photons having a frequency of $\omega_{0}$ of the SME (neglecting Lorentz-violating birefringence, which astrophysics experiments bound to $<10^{-37}$ [19], and a constant) [20]

$$
k=\omega_{0}\left[1-\frac{1}{2}\left(k_{F}\right)^{\alpha j \alpha k} \hat{k}_{j} \hat{k}_{k}-\left(k_{F}\right)^{\alpha 0 \alpha j} \hat{k}_{j}\right],
$$

where $k_{F}$ specifies Lorentz violation in the Maxwell sector. As $\vec{k}_{\text {eff }}=\vec{k}_{1}-\vec{k}_{2}$, where $\vec{k} \simeq \vec{k}_{1} \simeq-\vec{k}_{2}$, the last term cancels out. In our experiment the beams are vertical, $\hat{k}=\vec{k} / k=\hat{r}$. Thus, Eq. (11) reads

$$
\phi=2 k_{0} g_{0}\left[1+\frac{1}{2} \sigma^{j k} \hat{r}^{j} \hat{r}^{k}\right] T^{2}-\phi_{L} .
$$

where $g_{0}=G M / r^{2}$ and $k_{0}=\omega_{0} / c_{0}$ 21]. Thus, the measured anisotropy is given by $\sigma^{j k}=\bar{s}^{j k}-\left(k_{F}\right)^{\alpha j \alpha k}$. Various definitions of coordinates and fields can still be made, that could be chosen to yield $\left(k_{F}\right)^{\alpha j \alpha k}=0$.

By coordinate transformations from an inertial suncentered celestial equatorial frame (denoted with capital indices $J, K$ ) into the laboratory frame on Earth [18] we obtain the time-dependence

$$
\frac{\delta g}{g_{0}}=\sum_{m} C_{m} \cos \left(\omega_{m} t+\phi_{m}\right)+D_{m} \sin \left(\omega_{m} t+\phi_{m}\right)
$$

of the $g$-modulations. The coefficients $C_{m}, D_{m}$ for the six frequencies $\omega, 2 \omega, \omega \pm \Omega, 2 \omega \pm \Omega$ are functions of the components of $\sigma^{\mu \nu}$, of Earth's orbital velocity $v / c \simeq 10^{-4}$, and the frequencies of Earth's orbit $\Omega=2 \pi /(1 \mathrm{y})$ and rotation $\omega \simeq 2 \pi /(23.93 \mathrm{~h})$.

For bounding post-Newtonian gravity, we use $\sim 60 \mathrm{~h}$ of data taken with this setup, as well as a $\sim 60 \mathrm{~h}$ and a $\sim 10 \mathrm{~d}$ run reported previously [10], see Fig. 3. Periodic changes having an amplitude of around $100 \mu \mathrm{gal} \simeq 10^{-7} \mathrm{~g}$ are due to tides. Subtraction of a Newtonian model based on the relative positions of the Sun, the Moon, and the planets 22, 23] yields the graph shown at the bottom of Fig. 3. More sophisticated tidal models are available [24] that take into account ocean loading and local effects. However, such models typically rely, in part, on fitting $g$-observations and are thus not suitable for our purpose of comparing to a Newtonian model.

The combined data spans about $1500 \mathrm{~d}$, but fragmented into three relatively short segments. A Fourier

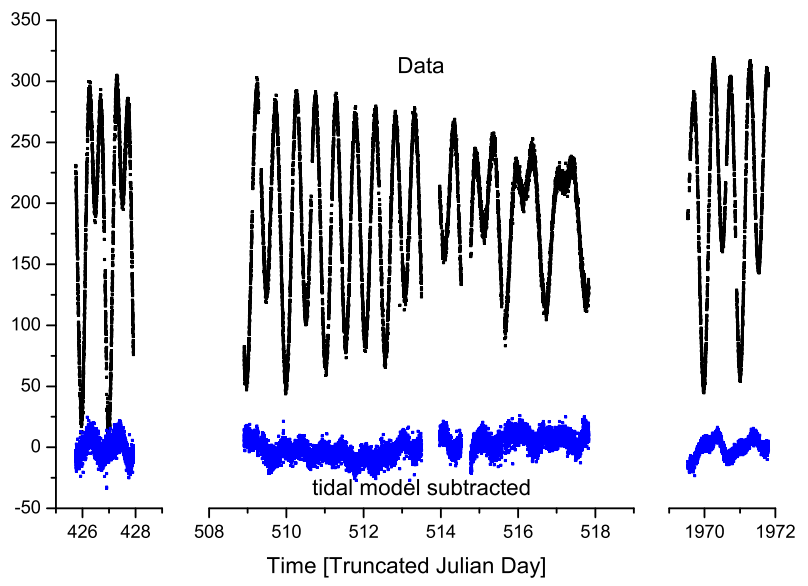

FIG. 3: Data in $10^{-9} \mathrm{~g}$. Each point represents a $60-\mathrm{s}$ scan of one fringe (75-s after TJD1900).

TABLE I: Results. Last column gives parts in $10^{9}$ for $\sigma^{J K}$ and parts in $10^{-5}$ for $\sigma^{T J}$.

\begin{tabular}{cccc}
\hline Comp. & Measured & Disentangled & Result \\
& $10^{-9}$ & $10^{-9}$ & \\
\hline$C_{2 \omega}$ & $0.342(88)$ & $-0.44(17)$ & $\sigma^{X X}-\sigma^{Y Y}=-5.6(2.1)$ \\
$D_{2 \omega}$ & $-0.942(89)$ & $-0.02(19)$ & $\sigma^{X Y}=-0.09(79)$ \\
$C_{\omega}$ & $3.668(88)$ & $3.1(8.8)$ & $\sigma^{X Z}=-13(37)$ \\
$D_{\omega}$ & $-0.267(85)$ & $14.8(9.0)$ & $\sigma^{Y Z}=-61(38)$ \\
$C_{2 \omega+\Omega}$ & $-1.378(87)$ & $-1.11(65)$ & $\sigma^{T Y}=172(100)$ \\
$D_{2 \omega+\Omega}$ & $-1.051(89)$ & $-1.08(68)$ & $\sigma^{T X}=-167(104)$ \\
$C_{2 \omega-\Omega}$ & $1.438(89)$ & $-0.30(66)$ & $\sigma^{T Y}=-2.0(4.4)$ \\
$D_{2 \omega-\Omega}$ & $0.536(88)$ & $0.82(67)$ & $\sigma^{T X}=5.4(4.5)$ \\
$C_{\omega+\Omega}$ & $0.647(94)$ & $-12.4(6.2)$ & $\sigma^{T X}=258(129)$ \\
$D_{\omega+\Omega}$ & $-2.020(82)$ & $-3.63(6.2)$ & $\sigma^{T Y}-0.21 \sigma^{T Z}=76(130)$ \\
$C_{\omega-\Omega}$ & $1.610(82)$ & $9.56(6.3)$ & $\sigma^{T X}=-200(130)$ \\
$D_{\omega-\Omega}$ & $2.840(92)$ & $0.11(6.1)$ & $\sigma^{T Z}+0.21 \sigma^{T Y}=-00(26)$ \\
\hline
\end{tabular}

analysis yields the components given in Tab. I The fragmentation of the data leads to significant overlap, as given by a covariance matrix cov. To remove the overlap, we form linear combinations using $\mathrm{cov}^{-1}$, see Tab. [1. The error in this estimate, given as the geometric sum of the errors entering the linear combinations, increases in this process.

Comparing the modulations of $g$ given by Eq. (6) to the measurement, we obtain the estimates listed in the fourth column of Tab. I. $\sigma^{T Z}$ is measured as a linear combination with $\sigma^{T Y}$, into which we insert $\sigma^{T Y}$ as previously determined. Some components are multiply determined and could be combined to a weighted average, but in all cases one limit strongly outweighs the others. Our final results are (parts in $10^{9}$ )

$$
\begin{array}{r}
\sigma^{X X}-\sigma^{Y Y}=-5.6(2.1), \quad \sigma^{X Y}=-0.09(79), \\
\sigma^{X Z}=-13(37), \quad \sigma^{Y Z}=-61(38)
\end{array}
$$

and (parts in $\left.10^{5}\right)$

$$
\begin{gathered}
\sigma^{T Y}=-2.0(4.4), \quad \sigma^{T X}=5.4(4.5), \\
\sigma^{T Z}=1.1(26) .
\end{gathered}
$$


In this letter, we have reported three types of results: First, a gravimeter based on cold atoms, which uses a pulse separation of $T=400 \mathrm{~ms}$ and a bright source of Cs atoms using Raman sideband cooling in an optical lattice to reach a resolution of $(8-11) \times 10^{-9} \mathrm{~g} / \sqrt{\mathrm{Hz}}$. Second, we analyze the expected modulation of the local gravitational acceleration apparent in this experiment as a result of Lorentz violation in both post-Newtonian gravity and electromagnetism. Third, our test of the isotropy of post-Newtonian gravity bounds four combinations of $\sigma^{J K}$ to the $10^{-9}$ level and the three $\sigma^{0 J}$ to the $10^{-5}$ level. Whereas most tests of gravity are astrophysical in nature [5], ours is a laboratory experiment, which offers reproducibility and superior control over relevant parameters.

A previous order-of-magnitude limit $\left|\bar{s}^{J K}\right| \leq 4 \times$ $10^{-9}$ exists, translated [18] from the anisotropic universe bounds due to Nordtvedt [4]. No such previous limits on the $\bar{s}^{T J}$ are known to us. A forthcoming publication derives bounds on $\bar{s}$ from 34 years of lunar laser ranging (LLR) data that complement our laboratory bounds [25]. We note, however, that ours is the first experiment where the simultaneous influence of the non-gravitational and gravitational effects are understood quantitatively and which accordingly states combined bounds. For other experiments, these influences are not understood at present. Moreover, the results differ vastly in the orbit (if one can think of the atoms' trajectory as an orbit) and quantummechanical nature of the test masses. This is interesting, as quantum gravity might conceivably involve phenomena that couple to coherent quantum states but not clas- sical objects.

Future bounds may be found by use of torsion balances, $g$-data that is routinely taken in geophysical research, or the gravity probe-B satellite. It is also interesting to study horizontal interferometer geometries, as they might offer suppression of tidal influences, which is the main factor limiting our resolution. In addition, lifting our assumption that UFF is valid, our data could be analyzed for bounds on $a$ - and $c$ - type SME matter coefficients [26]. We remark that gravity yields space-time varying contributions to $k_{F}$ related to Nambu-Goldstone modes [27]; our analysis uses a flat space-time picture where those are averaged over [18]. This is likely to yield higher-order corrections that are currently being investigated [26]. The gravimeter itself is still not limited by any fundamental limits such as quantum projection noise. With $\sim 10^{8}$ atoms per launch, a quantum projection limited gravimeter could reach the $10^{-12} \mathrm{~g}$ level per launch and $10^{-14} \mathrm{~g}$ per day, if other noise sources (notably phase noise and vibrations) can be controlled. This promises improved tests of gravity based on atom interferometry, deepening our understanding of the fundamental principles of Nature.

We thank A. Peters, Q.G. Bailey, J. Overduin, V.A. Kostelecky, and A. Mulch for valuable contributions. This material is based upon work supported by the National Science Foundation under Grant No. 0400866, the Air Force Office of Scientific Research, and the MultiUniversity Research Initiative.
[1] G. Amelino-Camelia et al. in: A.Macias, C. Lämmerzahl, and D. Nunez (eds.), Gravitation and Cosmology, page 30. AIP Conference Proceedings 758, Melville, N.Y. (2005).

[2] V.A. Kostelecky, Phys. Rev. D 69, 105009 (2004).

[3] D. Mattingly, Living Rev. Relativity 8, 5 (2005).

[4] K. Nordvedt, Phys. Rev. D 14, 1511 (1976).

[5] C.M. Will, Astrophys. J. 169, 141 (1971); Living Rev. Rel. 4, 4 (2001).

[6] K. Nordtvedt and C.M. Will, Astrophys. J. 177, 775 (1972).

[7] M. Kasevich and S. Chu, Phys. Rev. Lett. 67, 181 (1991).

[8] S. Chu, Nature (London) 416, 206 (2002).

[9] A. Wicht et al., Physica Scripta T102, 82 (2002); P. Clade et al., Phys. Rev. Lett. 96, 033001 (2006); H. Müller et al., Appl. Phys. B 84, 633-642 (2006).

[10] A. Peters, K.Y. Chung, and S. Chu, Nature 400, 849 (1999); Metrologia 38, 25 (2001).

[11] M.J. Snadden, J.M. McGuirk, P. Bouyer, K.G. Haritos, and M.A. Kasevich, Phys. Rev. Lett. 81, 971 (1998).

[12] T.L. Gustavson, A. Landragin, and M.A. Kasevich, Class. Quantum Gravity 17, 2385 (2000).

[13] J.B. Fixler, G.T. Foster, J.M. McGuirk, and M.A. Kasevich, Science 315, 74 (2007).

[14] S. Dimopoulos, P.W. Graham, J.M. Hogan, and M.A. Kasevich, Phys. Rev. Lett. 98, 111102 (2007).

[15] Kengyeow Chung, Ph.D. thesis, Stanford University
2001.

[16] J.M. Hensley, A. Peters, and S. Chu, Rev. Sci. Instrum. 70, 2735 (1999).

[17] P. Treutlein, K.Y. Chung, and S. Chu, Phys. Rev. A 63, 051401(r) (2001).

[18] Q.G. Bailey and V.A. Kostelecky, Phys. Rev. D 74, 045001 (2006).

[19] V.A. Kostelecký and M. Mewes, Phys. Rev. Lett. 97, 140401 (2006).

[20] V.A. Kostelecký and M. Mewes, Phys. Rev. D 66, 056005 (2002).

[21] $\omega_{0}$ is defined by spectroscopy of thermal atoms in a vapor cell; the stochastic orientation of their spins should suppress any sensitivity of $\omega$ to anisotropy.

[22] Y. Tamura, Bull. d'information marrées terrestres 99, 6813 (1987).

[23] Implemented using the ETGTAB v3.0 software by H.G. Wenzel, Univ. Karlsruhe.

[24] T.F. Baker and M.S. Bos, Geophys. J. Int. 152, 468 (2003).

[25] J.B.R. Battat, J.F. Chandler, and C.W. Stubbs, arXiv:0710.0702 1 (2007).

[26] V.A. Kostelecky and J.D. Tasson, to appear.

[27] R. Bluhm and V.A. Kostelecky, Phys. Rev. D 71, 065008 (2005). 